\title{
Theory of the Stark Effect on Single Molecular Spectroscopy
}

\author{
M. Hayashi ${ }^{\text {* }}$, C. H. Chianga ( 江建勳), K. K. Liang ${ }^{\mathrm{b}}$ (梁國淦), Y. J. Shiu ${ }^{\mathrm{b}}$ ( 許瑛珍 ), \\ C. H. Chang ${ }^{b}$ (張仲宏), D. Y. Wu ( 吳德印), F. Y. Li ( 李豐穎), \\ T.-C. Chang ${ }^{\mathrm{b}}$ (張大釗), U. Wild ${ }^{\mathrm{d}}$, T. Y. Latychevskaia ${ }^{\mathrm{d}}$ and S. H. Lin ${ }^{\mathrm{b}}$ (林聖賢) \\ ${ }^{a}$ Center for Condensed Matter Sciences, National Taiwan University, Taipei 106, Taiwan, R.O.C. \\ ${ }^{\mathrm{b}}$ Institute of Atomic and Molecular Sciences, Academia Sinica, Taipei, Taiwan 106, R.O.C. \\ ${ }^{\mathrm{c}}$ Department of Applied Chemistry, National Chi Nan University, Nantou 545, Taiwan, R.O.C. \\ ${ }^{\mathrm{d}}$ Swiss Federal Institute of Technology Zürich, Zürich, Switzerland
}

\begin{abstract}
In this work, we study a mechanism of the Stark effect on single molecular spectroscopy using the perturbation method. In particular, mechanisms of the induced dipole moment of a molecule imbedded in polar and non-polar host molecules are theoretically investigated by applying LCAO MO to a guest molecule. Ab initio calculations are performed to study how the distance between a guest and a host molecule affects the induced dipole and quadrapole moments. We have found that the induced dipole moment is very sensitive to short range configurations while the induced quadrapole moment can be affected by longer range configurations.
\end{abstract}

\section{INTRODUCTION}

The first Single-molecular Spectroscopy (SMS) experiment was performed in 1989 using absorption spectroscopy and a sophisticated modulation technique. ${ }^{1}$ Single molecules can be detected and manipulated in various environments. On solid surfaces, for instance, they can be studied using the scanning probe techniques of $\mathrm{STM}^{2}$ and SNOM. ${ }^{3}$ Single molecules can also be detected in liquids and in biological samples using fluorescence excitation. ${ }^{4}$ With the invention of SMS, the investigation of single dye molecules buried deep inside a transparent solid becomes feasible..$^{5-8}$

In the SMS literature, experiments on single molecules in electric fields are called the Stark effect. The first measurement on single molecules in an electric field were performed in 1992 by Wild et al. ${ }^{9}$ for the sample of pentacene in pterphenyl crystal. In the following years, the spectral shifts of single molecule resonances external electric fields were observed for various host/guest systems: ferrylene in polyethylene, ${ }^{10}$ perylene in n-nonane, ${ }^{11}$ terrylene in naphthaleln, ${ }^{12}$ dibenzanthanthrene in naphtalene and in HD matrices, ${ }^{13}$ etc.

According to the time-independent perturbation method, to the second-order approximation, the energy of the system can be expressed as

$$
E_{n}=E_{n}^{0}+H_{n n}^{\prime}+\sum_{m \neq n} \frac{\left|H_{n m}^{\prime}\right|^{2}}{E_{n}^{0}-E_{m}^{0}}+\cdots
$$

where $\hat{H}^{\prime}$ represents the perturbation. For the Stark effect, $\hat{H}^{\prime}$ is given by

$$
\hat{H}^{\prime}=-\vec{\mu} \cdot \vec{F}
$$

where $\vec{\mu}$ and $\vec{F}$ stand for the dipole operator and the applied electric field, respectively. Substituting Eq. (1-2) into Eq. (1-1) yields,

$$
E_{n}=E_{n}^{0}-\vec{\mu}_{n} \cdot \vec{F}-\frac{1}{2} \vec{F} \cdot \vec{\alpha}_{n} \cdot \vec{F}+\cdots
$$

where $\vec{\mu}_{n}$ represents the dipole moment $\vec{\mu}_{n} \equiv \vec{\mu}_{n n}=\left\langle\varphi_{n}^{0}|\vec{\mu}| \varphi_{n}^{0}\right\rangle$ and $\vec{\alpha}_{n}$ denotes the polarizability

$$
\vec{\alpha}_{n} \equiv \vec{\alpha}_{n n}=2 \sum_{m \neq n} \frac{\vec{\mu}_{m n} \otimes \vec{\mu}_{n m}}{E_{m}^{0}-E_{n}^{0}}
$$

Here $\vec{\mu}_{n m}$ is the transition dipole moment $\vec{\mu}_{n m}=\left\langle\varphi_{n}^{0}|\vec{\mu}| \varphi_{m}^{0}\right\rangle$. From Eq. (1-3), one can see that only when $\vec{\mu}_{n} \neq 0$ or $\vec{\mu}_{n} \cdot \vec{F} \neq$ 0 , can we observe the linear Stark effect. Since $\vec{\alpha}_{n} \neq 0$ for all systems, the second-order Stark effect can usually be observed provided that the field is strong enough.

Using Eq. (1-3), one can calculate the spectral shift due to the electric field as follows,

$$
\begin{aligned}
\Delta E_{n m}= & E_{n}-E_{m}=\left(E_{n}^{0}-E_{m}^{0}\right)-\left(\vec{\mu}_{n}-\vec{\mu}_{m}\right) \cdot \vec{F} \\
& -\frac{1}{2} \vec{F} \cdot\left(\vec{\alpha}_{n}-\vec{\alpha}_{m}\right) \cdot \vec{F}+\cdots
\end{aligned}
$$

In other words, the central problem in the linear Stark effect of SMS is the calculation of $\vec{\mu}_{n}$ and $\vec{\mu}_{m}$ induced by solvent molecules. 


\section{THEORETICAL}

\subsection{General Consideration}

We shall consider a case in which a non-polar molecule is dissolved in a solvent crystal. It should be noted that Wild group has observed the linear Stark effect in the fluorescence spectra of non-polar solute molecules in both polar and non-polar solvent matrices. This means that a dipole moment can be induced in the solute molecule by the polar or non-polar surrounding (or heat bath). In this section, we shall offer an interpretation for this phenomenon in terms of a molecular description based on the perturbation method.

Let us now consider a mechanism as to how a dipole moment can be induced by an interaction with environmental molecules. For this purpose, let $V$ describe the interaction between the solute molecule and solvent molecules. In this case, electronic wavefunctions of the solute molecule can be perturbed by $V$

$$
\left|\Phi_{g}\right\rangle=\left|\Phi_{g}^{0}\right\rangle+\sum_{e^{\prime} \neq g} \frac{\left\langle\Phi_{e^{\prime}}^{0}|V| \Phi_{g}^{0}\right\rangle}{E_{g}^{0}-E_{e^{\prime}}^{0}}\left|\Phi_{e^{\prime}}^{0}\right\rangle+\cdots
$$

and

$$
\left|\Phi_{e}\right\rangle=\left|\Phi_{e}^{0}\right\rangle+\sum_{e^{\prime \prime} \neq e} \frac{\left\langle\Phi_{e^{\prime \prime}}^{0}|V| \Phi_{e}^{0}\right\rangle}{E_{e}^{0}-E_{e^{\prime \prime}}^{0}}\left|\Phi_{e^{\prime \prime}}^{0}\right\rangle+\cdots
$$

where, for example, $\left|\Phi_{g}\right\rangle$ and $\left|\Phi_{e}\right\rangle$ denote the electronic wavefunctions of the ground and electronically excited states, respectively. The induced dipole-moments can be defined as

$$
\vec{\mu}_{g}^{\text {ind }}=\left\langle\Phi_{g}|\vec{\mu}| \Phi_{g}\right\rangle-\left\langle\Phi_{g}^{0}|\vec{\mu}| \Phi_{g}^{0}\right\rangle
$$

and

$$
\vec{\mu}_{e}^{i n d}=\left\langle\Phi_{e}|\vec{\mu}| \Phi_{e}\right\rangle-\left\langle\Phi_{e}^{0}|\vec{\mu}| \Phi_{e}^{0}\right\rangle
$$

Substituting Eqs. (2-1) and (2-2) into Eqs. (2-3) and (2-4), respectively, yield

$$
\vec{\mu}_{g}^{\text {ind }}=2 \sum_{e^{\prime} \neq g} \frac{\left\langle\Phi_{e^{\prime}}^{0}|V| \Phi_{g}^{0}\right\rangle}{E_{g}^{0}-E_{e^{\prime}}^{0}}\left\langle\Phi_{g}^{0}|\vec{\mu}| \Phi_{e^{\prime}}^{0}\right\rangle+\cdots
$$

and

$$
\vec{\mu}_{e}^{\text {ind }}=2 \sum_{e^{*} \neq e} \frac{\left\langle\Phi_{e^{\prime}}^{0}|V| \Phi_{e}^{0}\right\rangle}{E_{e}^{0}-E_{e^{\prime}}^{0}}\left\langle\Phi_{e}^{0}|\vec{\mu}| \Phi_{e^{\prime}}^{0}\right\rangle+\cdots
$$

\subsection{A non-polar molecule dissolved in polar molecules}

From Section 2.1, we can see that the central question in this problem is the calculation of $\left\langle\Phi_{e^{\prime}}^{0}|V| \Phi_{g}^{0}\right\rangle$ and $\left\langle\Phi_{e^{\prime \prime}}^{0}|V| \Phi_{e}^{0}\right\rangle$. In this section, we shall first consider the case of a non-polar molecule dissolved in polar molecules. The $V$ in this case is due to the dipole-electron interaction

$$
V=-\sum_{\alpha} \sum_{i} \frac{e\left(\vec{m}_{\alpha} \cdot \vec{R}_{\alpha i}\right)}{R_{\alpha i}^{3}}
$$

where $\vec{m}_{\alpha}$ denotes the dipole moment of AB, a solvent molecule and

$$
\vec{R}_{\alpha i}=\vec{R}_{0}+\vec{r}_{i}
$$

For $R_{\mathrm{o}} \gg r_{i}$, it follows that

$V=-e \sum_{0} \sum_{i}\left[\frac{\left(\vec{m}_{0} \cdot \vec{r}_{i}\right)}{R_{\alpha}^{3}}-\frac{3\left(\vec{m}_{\alpha} \cdot \vec{R}_{\alpha}\right)\left(\vec{R}_{0} \cdot \vec{r}_{i}\right)}{R_{0}^{5}}\right]+\cdots$

and that

$$
\left\langle\Phi_{e^{0}}^{0}|V| \Phi_{g}^{0}\right\rangle=\sum_{0}\left[\frac{\left(\vec{m}_{\alpha} \cdot \vec{\mu}_{e^{\prime} g}\right)}{R_{o}^{3}}-\frac{3\left(\vec{m}_{\alpha} \cdot \vec{R}_{\alpha}\right)\left(\vec{R}_{\alpha} \cdot \vec{\mu}_{e^{\prime} g}\right)}{R_{\alpha}^{5}}\right]+\cdots
$$

where

$$
\vec{\mu}_{e^{\prime} g}=\left\langle\Phi_{e^{\prime}}^{0}\left|-\sum_{i} e \vec{r}_{i}\right| \Phi_{g}^{0}\right\rangle .
$$

Similarly, we have

$$
\begin{aligned}
& \left\langle\Phi_{e^{\prime}}^{0}|V| \Phi_{e}^{0}\right\rangle= \\
& \quad \sum_{\alpha}\left[\frac{\left(\vec{m}_{\alpha} \cdot \vec{\mu}_{e^{\prime} e}\right)}{R_{\alpha}^{3}}-\frac{3\left(\vec{m}_{\alpha} \cdot \vec{R}_{o}\right)\left(\vec{R}_{\alpha} \cdot \vec{\mu}_{e^{\prime} e}\right)}{R_{o}^{5}}\right]+\cdots
\end{aligned}
$$

\subsection{A non-polar molecule dissolved in a non-polar crystal}

In this section, we shall consider the case of a non-polar molecule dissolved in a non-polar crystal. Suppose that we let A represent the solute molecule while we let $\mathrm{D}$ represent a solvent molecule. Then the interaction between them can be written by

$$
V=-\sum_{D}\left(e^{2} \sum_{i} \frac{\vec{r}_{D_{i}} \cdot \vec{\alpha}_{D} \cdot \vec{r}_{D_{i}}}{2 r_{D_{i}}^{6}}\right)
$$

where $\vec{\alpha}_{D}$ denotes the polarizability tensor of $\mathrm{D}$,

$$
\vec{\alpha}_{D}=2 \sum_{n^{\prime}} \frac{\vec{\mu}_{n^{\prime} n} \otimes \vec{\mu}_{n n^{\prime}}}{E_{n}-E_{n}}
$$

Here $\vec{\mu}_{n^{\prime} n}$ is the transition moment of D.

For an isotropic D molecule, we notice that $\vec{\alpha}_{D} \rightarrow \alpha_{D} \vec{I}$ and Eq. (4-1) becomes

where

$$
V=-\sum_{D} \sum_{i} \frac{\alpha_{D} e^{2}}{2 r_{D i}^{4}}
$$

$$
\frac{1}{r_{D i}^{4}}=\frac{1}{R_{D A}^{4}}-\frac{4\left(\vec{R}_{D A} \cdot \vec{r}_{A i}\right)}{R_{D A}^{6}}+\cdots
$$


It follows that

$$
\begin{aligned}
& \left\langle\Phi_{e^{\prime}}^{0}|V| \Phi_{g}^{0}\right\rangle=-\sum_{D} \frac{2 \alpha_{D}\left(\vec{R}_{D A} \cdot \vec{\mu}_{e^{\prime} g}\right)}{R_{D A}^{6}}+\cdots \\
& \left\langle\Phi_{e}^{0}|V| \Phi_{g}^{0}\right\rangle=-\sum_{D} \frac{2 \alpha_{D}\left(\vec{R}_{D A} \cdot \vec{\mu}_{e g}\right)}{R_{D A}^{6}}+\cdots
\end{aligned}
$$

and

$$
\left\langle\Phi_{e}^{0}|V| \Phi_{g}^{0}\right\rangle=-\sum_{D} \frac{2 \alpha_{D}\left(\vec{R}_{D A} \cdot \vec{\mu}_{e^{\prime} g}\right)}{R_{D A}^{6}}+\cdots .
$$

Here the sum over D refers to the summation over the solvent molecules.

In conclusion, in this section based on the use of the perturbation method we propose a theory of linear Stark effect for non-polar molecules dissolved in a polar matrix and a non-polar matrix. For the latter, the induced dipole moment arises from the asymmetric orientation of the solvent motion in the solvent cage and/or from the structure asymmetry of the solvent cage.

\section{APPLICATION}

\section{LCAO MO method}

In this section, we shall show how induced dipole moments can be expressed in terms of molecular orbital theory. For simplicity, let us apply the theoretical results to the B electrons of butadiene. Notice that the LCAO-MO method gives us

$$
\begin{aligned}
& W_{1}=\alpha+\frac{(\sqrt{5}+1) \beta}{2} \\
& \chi_{1}=\frac{1}{\sqrt{5+\sqrt{5}}}\left[\phi_{a}+\frac{(\sqrt{5}+1)}{2} \phi_{b}+\frac{(\sqrt{5}+1)}{2} \phi_{c}+\phi_{d}\right] \\
& W_{2}=\alpha+\frac{(\sqrt{5}-1) \beta}{2} \\
& \chi_{2}=\frac{1}{\sqrt{5-\sqrt{5}}}\left[\phi_{a}+\frac{(\sqrt{5}-1)}{2} \phi_{b}-\frac{(\sqrt{5}-1)}{2} \phi_{c}-\phi_{d}\right] \\
& W_{3}=\alpha-\frac{(\sqrt{5}-1) \beta}{2} \\
& \chi_{3}=\frac{1}{\sqrt{5-\sqrt{5}}}\left[\phi_{a}-\frac{(\sqrt{5}-1)}{2} \phi_{b}-\frac{(\sqrt{5}-1)}{2} \phi_{c}+\phi_{d}\right] \\
& W_{4}=\alpha-\frac{(\sqrt{5}+1) \beta}{2}
\end{aligned}
$$

and

$$
\chi_{4}=\frac{1}{\sqrt{5+\sqrt{5}}}\left[\phi_{a}-\frac{(\sqrt{5}+1)}{2} \phi_{b}+\frac{(\sqrt{5}+1)}{2} \phi_{c}-\phi_{d}\right]
$$

where $\left\{\phi_{i}\right\}$ and $\left\{W_{i}\right\}$ represent the atomic orbitals and orbital energies, respectively. It follows that

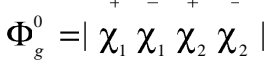

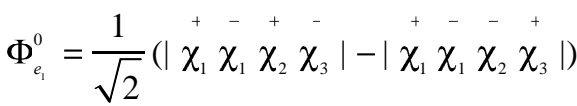

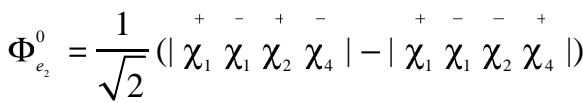

where, for example, $\left|+\bar{\chi}_{1}-++-\overline{\chi_{1}} \bar{\chi}_{2} \bar{\chi}_{2}\right|$ denotes the Slater determinant. The energy of each state corresponding to Eqs. (5-9)(5-11) can be, respectively, given by

$$
\begin{aligned}
& E_{g}^{0}=2 W_{1}+2 W_{2} \\
& E_{e_{1}}^{0}=2 W_{1}+W_{2}+W_{3}
\end{aligned}
$$

and

$$
E_{e_{2}}^{0}=2 W_{1}+W_{2}+W_{4}
$$

Using Eqs. (5-9)-(5-11), we obtain

$$
\begin{aligned}
\vec{\mu}_{g}^{\text {ind }}= & 2 \sum_{s \neq g} \frac{\left\langle\Phi_{s}^{0}|V| \Phi_{g}^{0}\right\rangle}{E_{g}^{0}-E_{s}^{0}}\left\langle\Phi_{g}^{0}|\vec{\mu}| \Phi_{s}^{0}\right\rangle+\cdots \\
= & 2 \frac{\left\langle\Phi_{e_{1}}^{0}|V| \Phi_{g}^{0}\right\rangle}{E_{g}^{0}-E_{e_{1}}^{0}}\left\langle\Phi_{g}^{0}|\vec{\mu}| \Phi_{e_{1}}^{0}\right\rangle \\
& +2 \frac{\left\langle\Phi_{e_{2}}^{0}|V| \Phi_{g}^{0}\right\rangle}{E_{g}^{0}-E_{e_{2}}^{0}}\left\langle\Phi_{g}^{0}|\vec{\mu}| \Phi_{e_{2}}^{0}\right\rangle \\
\vec{\mu}_{e_{1}}^{\text {ind }}= & 2 \sum_{s \neq e_{1}} \frac{\left\langle\Phi_{s}^{0}|V| \Phi_{e_{1}}^{0}\right\rangle}{E_{e_{1}}^{0}-E_{s}^{0}}\left\langle\Phi_{e_{1}}^{0}|\vec{\mu}| \Phi_{s}^{0}\right\rangle+\cdots \\
= & 2 \frac{\left\langle\Phi_{g}^{0}|V| \Phi_{e_{1}}^{0}\right\rangle}{E_{e_{1}}^{0}-E_{g}^{0}}\left\langle\Phi_{e_{1}}^{0}|\vec{\mu}| \Phi_{g}^{0}\right\rangle \\
& +2 \frac{\left\langle\Phi_{e_{2}}^{0}|V| \Phi_{e_{1}}^{0}\right\rangle}{E_{e_{1}}^{0}-E_{e_{2}}^{0}}\left\langle\Phi_{e_{1}}^{0}|\vec{\mu}| \Phi_{e_{2}}^{0}\right\rangle
\end{aligned}
$$

and 


$$
\begin{aligned}
\Delta \vec{\mu}_{e_{1} g}^{\text {ind }}= & \vec{\mu}_{e_{1}}^{\text {ind }}-\vec{\mu}_{g}^{\text {ind }} \\
= & 4 \frac{\left\langle\Phi_{g}^{0}|V| \Phi_{e_{1}}^{0}\right\rangle}{E_{e_{1}}^{0}-E_{g}^{0}}\left\langle\Phi_{e_{1}}^{0}|\vec{\mu}| \Phi_{g}^{0}\right\rangle \\
& +2 \frac{\left\langle\Phi_{e_{2}}^{0}|V| \Phi_{g}^{0}\right\rangle}{E_{g}^{0}-E_{e_{2}}^{0}}\left\langle\Phi_{g}^{0}|\vec{\mu}| \Phi_{e_{2}}^{0}\right\rangle \\
& +2 \frac{\left\langle\Phi_{e_{2}}^{0}|V| \Phi_{e_{1}}^{0}\right\rangle}{E_{e_{1}}^{0}-E_{e_{2}}^{0}}\left\langle\Phi_{e_{1}}^{0}|\vec{\mu}| \Phi_{e_{2}}^{0}\right\rangle
\end{aligned}
$$

or

$$
\begin{aligned}
& \Delta \vec{\mu}_{e_{1} g}^{\text {ind }}=\vec{\mu}_{e_{1}}^{i n d}-\vec{\mu}_{g}^{\text {ind }} \\
& =4 \frac{\left\langle\Phi_{g}^{0}|V| \Phi_{e_{1}}^{0}\right\rangle}{W_{3}-W_{2}}\left\langle\Phi_{e_{1}}^{0}|\vec{\mu}| \Phi_{g}^{0}\right\rangle \\
& +2 \frac{\left\langle\Phi_{e_{2}}^{0}|V| \Phi_{g}^{0}\right\rangle}{W_{2}-W_{4}}\left\langle\Phi_{g}^{0}|\vec{\mu}| \Phi_{e_{2}}^{0}\right\rangle \\
& +2 \frac{\left\langle\Phi_{e_{2}}^{0}|V| \Phi_{e_{1}}^{0}\right\rangle}{W_{3}-W_{4}}\left\langle\Phi_{e_{1}}^{0}|\vec{\mu}| \Phi_{e_{2}}^{0}\right\rangle
\end{aligned}
$$

or

$$
\begin{aligned}
& \Delta \vec{\mu}_{e_{1} g}^{i n d}=\vec{\mu}_{e_{1}}^{i n d}-\vec{\mu}_{g}^{i n d} \\
& =4 \frac{\sqrt{2}\left\langle\chi_{2}|V| \chi_{3}\right\rangle}{W_{3}-W_{2}} \sqrt{2}\left\langle\chi_{3}|\vec{\mu}| \chi_{2}\right\rangle \\
& +2 \frac{\sqrt{2}\left\langle\chi_{4}|V| \chi_{2}\right\rangle}{W_{2}-W_{4}} \sqrt{2}\left\langle\chi_{2}|\vec{\mu}| \chi_{4}\right\rangle \\
& +2 \frac{\left\langle\chi_{4}|V| \chi_{3}\right\rangle}{W_{3}-W_{4}}\left\langle\chi_{3}|\vec{\mu}| \chi_{4}\right\rangle
\end{aligned}
$$

Substituting the dipole moments and energies yields (see Appendices $\mathrm{A}$ and $\mathrm{B}$ )

$$
\begin{aligned}
& \Delta \vec{\mu}_{e_{1} g}^{\text {ind }}=\vec{\mu}_{e_{1}}^{\text {ind }}-\vec{\mu}_{g}^{\text {ind }} \\
& =4 \frac{\sqrt{2}\left\langle\chi_{2}|V| \chi_{3}\right\rangle}{-(\sqrt{5}-1) \beta} \frac{(3+\sqrt{5})}{\sqrt{2}(5-\sqrt{5})}\left(e R_{b a}\right) \\
& +2 \frac{\left\langle\chi_{4}|V| \chi_{3}\right\rangle}{\beta} \frac{2}{\sqrt{5}}\left(e R_{b a}\right)
\end{aligned}
$$

For a non-polar molecule dissolved in polar molecules, Eq. (5-17) becomes (see Appendix C)

$$
\Delta \vec{\mu}_{e_{1} g}^{i n d}=\vec{\mu}_{e_{1}}^{i n d}-\vec{\mu}_{g}^{i n d}=C\left(\frac{e \vec{R}_{b a}}{\beta}\right) \sum_{0} V_{\vec{m}}\left(R_{\alpha}\right)
$$

where

$$
C=\frac{4}{(1-\sqrt{5})}\left(\frac{3+\sqrt{5}}{\sqrt{2}(5-\sqrt{5})}\right)^{2}+\frac{8}{5}
$$

$$
V_{\vec{m}}\left(R_{\alpha}\right)=\left[\frac{\vec{m}_{0} \cdot\left(e \vec{R}_{b a}\right)}{R_{\alpha}^{3}}-\frac{3\left(\vec{m}_{0} \cdot \vec{R}_{0}\right)\left\{\vec{R}_{\alpha} \cdot\left(e \vec{R}_{b a}\right)\right\}}{R_{\alpha}^{5}}\right]
$$

On the other hand, for a non-polar molecule dissolved in a non-polar crystal, we obtain

$$
\Delta \vec{\mu}_{e_{1} g}^{i n d}=\vec{\mu}_{e_{1}}^{i n d}-\vec{\mu}_{g}^{i n d}=-C\left(\frac{e \vec{R}_{b a}}{\beta}\right) \sum_{D} V_{\alpha_{D}}\left(\vec{R}_{D A}\right)
$$

where

$$
V_{\alpha_{D}}\left(\vec{R}_{D A}\right)=\frac{2 \alpha_{D}\left\{\vec{R}_{D A} \cdot\left(e \vec{R}_{b a}\right)\right\}}{R_{D A}^{6}}
$$

Given the molecular properties for each case, one can roughly estimate the difference of induced dipole moment by using Eq. (5-19) or Eq. (5-22).

\section{Ab initio study}

In the previous section, we have shown, using LCAO MO, molecular mechanisms of the induced dipole moment for the two cases: (1) a non-polar molecule dissolved in polar molecules and (2) a non-polar molecule dissolved in a non-polar crystal. In this section, to understand the induced dipole and induced quadruple moments more realistically, we shall apply the ab inito calculation method to compute these induced moments of a model system. For this purpose, we consider a model system consisting of a ethylene molecule and a rare gas molecule. This model is one of the simplest models for a non-polar molecule dissolved in a non-polar crystal. For the calculation method, we employ the Gaussian package ${ }^{14}$ and apply DFT with B3YKLP and 6-311+g** basis set to the ground state and that CIS with the same basis set to the excited state. The main focus in this computation is to present the induced moment as a function of the distance between the ethylene and the rare gas molecule. Table 1 lists the calculated dipole moments of the ethylene and He system as a function of the distance between the two molecules. Fig. 1, for example, shows a geometry used for the calculation of ethylene and $\mathrm{He}$

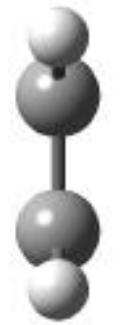

Side view

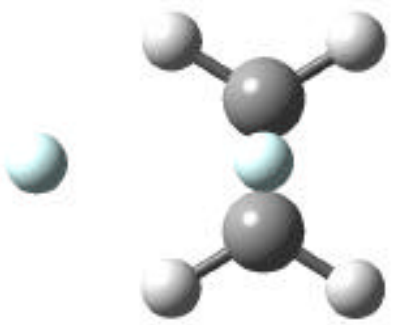

Top view
Fig. 1. A geometry used for the calculation of ethylene and $\mathrm{He}$ at a distance of $3 \AA$. 
at a distance of $3 \AA$. Table 2 lists the calculated induced quadrapole moment of this system.

Fig. 2 shows the calculated induced dipole and quadrapole moments for ethylene+He, ethylene+Ne, and ethylene+Ar as a function of the distance between ethylene and a

Table 1. Dipole Moment of Ethylene $+\mathrm{He}$

\begin{tabular}{lccccccc}
\hline & \multicolumn{3}{c}{ Ground state } & & \multicolumn{3}{c}{ Excited state } \\
\cline { 2 - 5 } \cline { 6 - 8 } Distance & $\mu_{\mathrm{x}}$ & $\mu_{\mathrm{y}}$ & $\mu_{\mathrm{z}}$ & & $\mu_{\mathrm{x}}$ & $\mu_{\mathrm{y}}$ & $\mu_{\mathrm{z}}$ \\
1 & 0 & 0 & 1.0145 & & 0 & 0 & 3.7543 \\
2 & 0 & 0 & 0.4721 & & 0 & 0 & 0.541 \\
3 & 0 & 0 & 0.0843 & & 0 & 0 & 0.0888 \\
4 & 0 & 0 & 0.0214 & & 0 & 0 & 0.0133 \\
5 & 0 & 0 & 0.0064 & & 0 & 0 & 0.0022 \\
6 & 0 & 0 & 0.0006 & & 0 & 0 & -0.0002 \\
$\infty$ & 0 & 0 & 0 & & 0 & 0 & 0 \\
\hline
\end{tabular}

In units of Debye
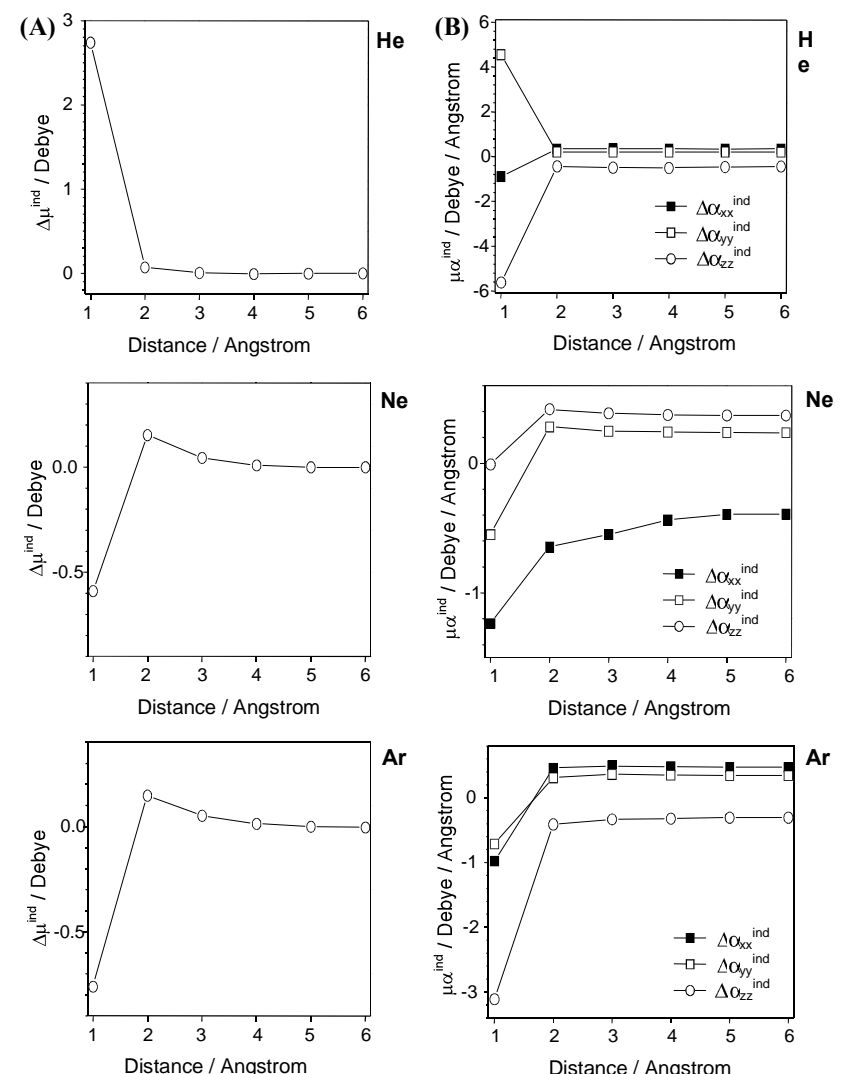

Fig. 2. The calculated induced moments for ethylene $+\mathrm{He}$, ethylene $+\mathrm{Ne}$, and ethylene + Ar. The results; (A) induced dipole moments and (B) induced quadrapole moments, are shown as a function of the distance between ethylene and a rare gas atom. rare gas atom. From Fig. 2A, one can see that the induced dipole moment of the ethylene+He system quickly decreases as the distance increases larger than $1 \AA$ and it becomes almost zero with the distance larger than $2 \AA$. Within $2 \AA$ similar behavior can be seen in the $\mathrm{xx}$ and yy components of the induced quadrapole moment of the same system. However, the zz component of the induced quadrapole moment cannot become zero within $6 \AA$. It is interesting to see other systems like the ethylene+Ne and ethylene+Ar systems. The results are listed in Tables 3-6. The middle and last panels of Fig. 2 show the induced dipole and quadrapole moments of these systems. A quick change of the amplitude of the induced di-

Table 2. Quadrapole Moment of Ethylene $+\mathrm{He}$

\begin{tabular}{lccccccc}
\hline & \multicolumn{3}{c}{ Ground state } & & \multicolumn{3}{c}{ Excited state } \\
\cline { 2 - 3 } \cline { 6 - 8 } Distance & $\alpha_{\mathrm{xx}}$ & $\alpha_{\mathrm{yy}}$ & $\alpha_{\mathrm{zz}}$ & & $\alpha_{\mathrm{xx}}$ & $\alpha_{\mathrm{yy}}$ & $\alpha_{\mathrm{zz}}$ \\
\hline 1 & -13.751 & -18.190 & -18.037 & & -14.911 & -13.605 & -23.464 \\
2 & -13.558 & -13.576 & -16.993 & & -13.48 & -13.342 & -17.230 \\
3 & -13.543 & -13.503 & -16.879 & & -13.461 & -13.262 & -17.172 \\
4 & -13.532 & -13.483 & -16.893 & -13.460 & -13.250 & -17.195 \\
5 & -13.526 & -13.476 & -16.95 & & -13.460 & -13.249 & -17.215 \\
6 & -13.524 & -13.474 & -16.982 & -13.459 & -13.248 & -17.230 \\
$\infty$ & -15.903 & -12.434 & -12.389 & -16.171 & -12.404 & -12.193 \\
\hline
\end{tabular}

In units of Debye-Angstrom; $\alpha_{x y}=\alpha_{x z}=\alpha_{y z}=0$.

Table 3. Dipole Moment of Ethylene $+\mathrm{Ne}$

\begin{tabular}{lccccccc}
\hline & \multicolumn{3}{c}{ Ground state } & & \multicolumn{3}{c}{ Excited state } \\
\cline { 2 - 5 } \cline { 6 - 8 } Distance & $\mu_{\mathrm{x}}$ & $\mu_{\mathrm{y}}$ & $\mu_{\mathrm{z}}$ & & $\mu_{\mathrm{x}}$ & $\mu_{\mathrm{y}}$ & $\mu_{\mathrm{z}}$ \\
\hline 1 & 0 & 0 & -0.9472 & & 0 & 0 & -1.5365 \\
2 & 0 & 0 & 0.5848 & & 0 & 0 & 0.7364 \\
3 & 0 & 0 & 0.0738 & & 0 & 0 & 0.1172 \\
4 & 0 & 0 & 0.004 & & 0 & 0 & 0.0114 \\
5 & 0 & 0 & 0.0014 & & 0 & 0 & 0.0009 \\
6 & 0 & 0 & 0.0002 & & 0 & 0 & -0.0006 \\
$\infty$ & 0 & 0 & 0 & 0 & 0 & 0 \\
\hline
\end{tabular}

In units of Debye

Table 4. Quadrapole Moment of Ethylene + Ne

\begin{tabular}{lccccccc}
\hline & \multicolumn{3}{c}{ Ground state } & & \multicolumn{3}{c}{ Excited state } \\
\cline { 2 - 3 } \cline { 6 - 8 } Distance & $\alpha_{\mathrm{xx}}$ & $\alpha_{\mathrm{yy}}$ & $\alpha_{\mathrm{zz}}$ & & $\alpha_{\mathrm{xx}}$ & $\alpha_{\mathrm{yy}}$ & $\alpha_{\mathrm{zz}}$ \\
\hline 1 & -22.554 & -21.290 & -32.100 & & -23.800 & -21.972 & -35.013 \\
2 & -16.913 & -17.063 & -21.119 & & -16.723 & -16.723 & -21.334 \\
3 & -16.862 & -16.840 & -20.382 & & -16.637 & -16.446 & -20.519 \\
4 & -16.828 & -16.785 & -20.277 & -16.618 & -16.405 & -20.402 \\
5 & -16.825 & -16.782 & -20.280 & -16.62 & -16.408 & -20.391 \\
6 & -16.824 & -16.782 & -20.283 & -16.619 & -16.409 & -20.392 \\
$\infty$ & -15.903 & -12.434 & -12.389 & -16.171 & -12.404 & -12.193 \\
\hline \multicolumn{2}{l}{ In units of Debye-Angstrom; $\alpha_{\mathrm{xy}}=\alpha_{\mathrm{xz}}=\alpha_{\mathrm{yz}}=0}$.
\end{tabular}


Table 5. Dipole Moment of Ethylene $+\mathrm{Ar}$

\begin{tabular}{lccccccc}
\hline & \multicolumn{3}{c}{ Ground state } & & \multicolumn{3}{c}{ Excited state } \\
\cline { 2 - 5 } \cline { 6 - 8 } Distance & $\mu_{\mathrm{x}}$ & $\mu_{\mathrm{y}}$ & $\mu_{\mathrm{z}}$ & & $\mu_{\mathrm{x}}$ & $\mu_{\mathrm{y}}$ & $\mu_{\mathrm{z}}$ \\
\hline 1 & 0 & 0 & -3.4808 & & 0 & 0 & -4.242 \\
2 & 0 & 0 & 0.6714 & & 0 & 0 & 0.8180 \\
3 & 0 & 0 & 0.053 & & 0 & 0 & 0.1038 \\
4 & 0 & 0 & -0.0136 & & 0 & 0 & -0.0005 \\
5 & 0 & 0 & -0.0058 & & 0 & 0 & -0.0066 \\
6 & 0 & 0 & 0.0002 & & 0 & 0 & -0.0043 \\
$\infty$ & 0 & 0 & -0.0031 & & 0 & 0 & 0 \\
\hline
\end{tabular}

In units of Debye

Table 6. Quadrapole Moment of Ethylene $+\mathrm{Ar}$

\begin{tabular}{lccccccc}
\hline & \multicolumn{3}{c}{ Ground state } & & \multicolumn{3}{c}{ Excited state } \\
\cline { 2 - 5 } \cline { 6 - 8 } Distance & $\alpha_{\mathrm{xx}}$ & $\alpha_{\mathrm{yy}}$ & $\alpha_{\mathrm{zz}}$ & & $\alpha_{\mathrm{xx}}$ & $\alpha_{\mathrm{yy}}$ & $\alpha_{\mathrm{zz}}$ \\
\hline 1 & -27.206 & -45.262 & -31.914 & & -27.482 & -45.781 & -32.954 \\
2 & -24.057 & -24.248 & -31.566 & -23.908 & -23.936 & -32.015 \\
3 & -24.217 & -24.199 & -28.417 & & -24.100 & -23.922 & -28.771 \\
4 & -24.193 & -24.149 & -27.799 & -24.087 & -23.878 & -28.041 \\
5 & -24.186 & -24.140 & -27.700 & -24.084 & -23.872 & -27.899 \\
6 & -24.186 & -24.141 & -27.682 & -24.085 & -23.874 & -27.877 \\
$\infty$ & -15.903 & -12.434 & -12.389 & -16.171 & -12.404 & -12.193 \\
\hline
\end{tabular}

In units of Debye-Angstrom; $\alpha_{x y}=\alpha_{x z}=\alpha_{y z}=0$.

pole moments can also be seen in these two systems, but it takes a longer distance to become zero. The induced quadrapole moments also show large changes within $2 \AA$ and the zz-components still keep large values even at $6 \AA$.

The above calculations indicate that a short range configuration of hosts can affect the induced dipole moment of a guest molecule and relatively longer range configurations can affect the quadrapole of the guest molecule.

In summary, we have theoretically shown, using the perturbation method, a mechanism of the induced dipole moment of a molecule imbedded in polar and non-polar host molecules. To roughly estimate the effect of induced dipole moment, we have applied LCAO MO to a guest molecule. We have also performed ab initio calculations to study how the distance between a guest and a host molecule affects the induced dipole and quadrapole moments. We have found that the induced dipole moment is very sensitive to short range configurations and, on the other hand, the induced quadrapole moment can be affected by longer range configurations.

\section{ACKNOWLEDGEMENTS}

We wish to thank the National Science Council (NSC) of the ROC and Academia Sinica for financial support of this work. MH and CHC would also like to thank the National Center for High-Performance Computing for Gaussian calculation support. $\mathrm{MH}$ would like to thank NSC of the ROC for financial support (NSC-90-2113-M-002-024).

Received June 28, 2002.

\section{Key Words}

Stark effect; Single molecular spectroscopy;

Polarizability; Dipole moment.

\section{Appendix A. Matrix elements in atomic orbitals}

We need to calculate the matrix elements $\left\langle\Phi_{e_{1}}^{0}|V| \Phi_{g}^{0}\right\rangle$, $\left\langle\Phi_{e 2}^{0}|V| \Phi_{g}^{0}\right\rangle,\left\langle\Phi_{e_{2}}^{0}|V| \Phi_{e_{1}}^{0}\right\rangle,\left\langle\Phi_{g}^{0}|\vec{\mu}| \Phi_{e_{1}}^{0}\right\rangle$ and $\left\langle\Phi_{g}^{0}|\vec{\mu}| \Phi_{e_{2}}^{0}\right\rangle$. Let us start with calculation of $\left\langle\Phi_{e_{1}}^{0}|V| \Phi_{g}^{0}\right\rangle$. Using Eqs. (5-9) and (510), we have

$$
\begin{aligned}
& \left\langle\Phi_{e_{1}}^{0}|V| \Phi_{g}^{0}\right\rangle
\end{aligned}
$$

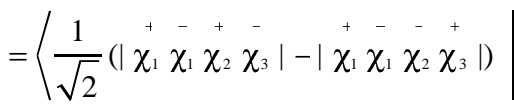

$$
\begin{aligned}
& =\frac{1}{\sqrt{2}}\left\langle\left|\begin{array}{cccc}
\mid+ & + & - \\
\chi_{1} & \chi_{1} & \chi_{2} & \chi_{3}
\end{array}\right||V|\left|\begin{array}{llll}
+ & - & + \\
\chi_{1} & \chi_{1} & \chi_{2} & \chi_{2}
\end{array}\right|\right\rangle
\end{aligned}
$$

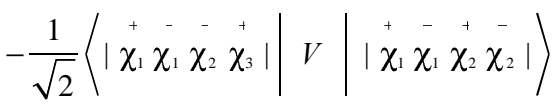

$$
\begin{aligned}
& =\frac{1}{\sqrt{2}}\left\langle\begin{array}{cccc}
+ & - & - \\
\chi_{1} & \chi_{1} & \chi_{2} & \chi_{3}
\end{array}|| V\left|\begin{array}{cccc}
+ & - & + \\
\chi_{1} & \chi_{1} & \chi_{2} & \chi_{2}
\end{array}\right|\right\rangle
\end{aligned}
$$

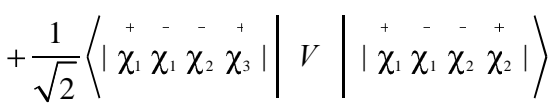

$$
\begin{aligned}
& =\frac{2}{\sqrt{2}}\left\langle\chi_{3}|V| \chi_{2}\right\rangle=\sqrt{2}\left\langle\chi_{3}|V| \chi_{2}\right\rangle
\end{aligned}
$$

In similar fashion, we obtain

$$
\begin{aligned}
& \left\langle\Phi_{e_{2}}^{0}|V| \Phi_{g}^{0}\right\rangle
\end{aligned}
$$

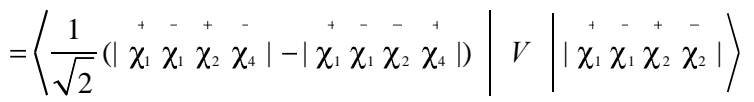

$$
\begin{aligned}
& =\frac{1}{\sqrt{2}}\left\langle\begin{array}{cccc}
\mid+ & - & + \\
\chi_{1} & \chi_{1} & \chi_{2} & \chi_{4}
\end{array}|| V|| \begin{array}{cccc}
+ & - & + & - \\
\chi_{1} & \chi_{1} & \chi_{2} & \chi_{2}
\end{array} \mid\right\rangle
\end{aligned}
$$

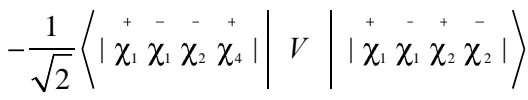

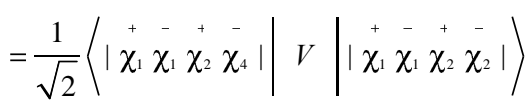

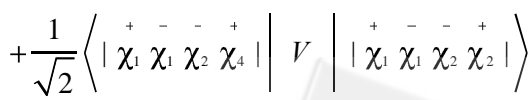

$$
\begin{aligned}
& =\sqrt{2}\left\langle\chi_{4}|V| \chi_{2}\right\rangle
\end{aligned}
$$


and

$$
\begin{aligned}
& \left\langle\Phi_{e_{1}}^{0}|V| \Phi_{e_{2}}^{0}\right\rangle
\end{aligned}
$$

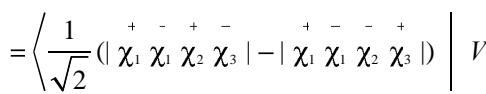

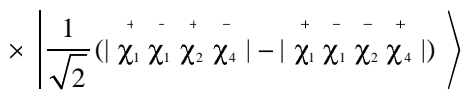

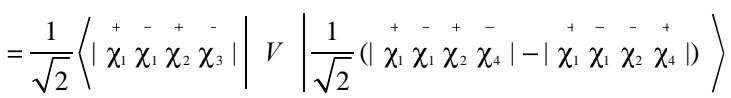

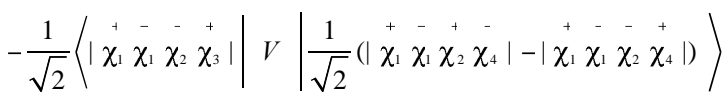

$$
\begin{aligned}
& =\frac{1}{2}\left\langle\left|\begin{array}{llll}
\mid & - & + & - \\
\chi_{1} & \chi_{1} & \chi_{2} & \chi_{3}
\end{array}\right||V| \begin{array}{cccc}
+ & - & + \\
\chi_{1} & \chi_{1} & \chi_{2} & \chi_{4}
\end{array} \mid\right\rangle
\end{aligned}
$$

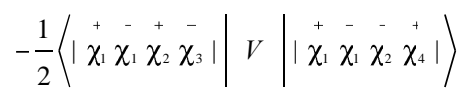

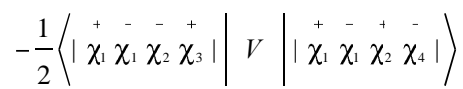

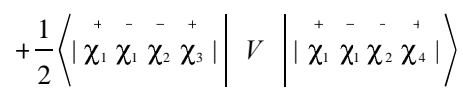

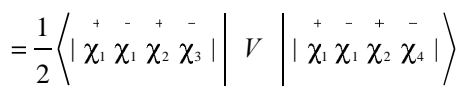

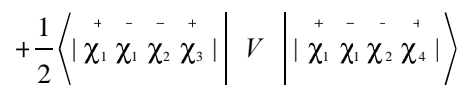

$$
\begin{aligned}
& =\left\langle\chi_{3}|V| \chi_{4}\right\rangle
\end{aligned}
$$

It is obvious from Eqs. (A-1)-(A-3) that

$$
\begin{aligned}
& \left\langle\Phi_{g}^{0}|\vec{\mu}| \Phi_{e_{1}}^{0}\right\rangle=\sqrt{2}\left\langle\chi_{2}|\vec{\mu}| \chi_{3}\right\rangle=\sqrt{2} \vec{\mu}_{23} \\
& \left\langle\Phi_{g}^{0}|\vec{\mu}| \Phi_{e_{2}}^{0}\right\rangle=\sqrt{2}\left\langle\chi_{4}|\vec{\mu}| \chi_{2}\right\rangle=\sqrt{2} \vec{\mu}_{42}
\end{aligned}
$$

and

$$
\left\langle\Phi_{e_{1}}^{0}|\vec{\mu}| \Phi_{e_{2}}^{0}\right\rangle=\left\langle\chi_{3}|\vec{\mu}| \chi_{4}\right\rangle=\vec{\mu}_{34}
$$

\section{Appendix B. Calculation of $\vec{\mu}_{23}, \vec{\mu}_{42}, \vec{\mu}_{34}$}

We begin with $\vec{\mu}_{23}$

$\left\langle\Phi_{s}^{0}|\vec{\mu}| \Phi_{c_{1}}^{0}\right\rangle=\sqrt{2}\left\langle\chi_{2}|\vec{\mu}| \chi_{3}\right\rangle=\sqrt{2} \vec{\mu}_{23}$

$$
\begin{aligned}
= & \sqrt{2}\left\langle\frac{1}{\sqrt{5-\sqrt{5}}}\left[\phi_{a}+\frac{(\sqrt{5}-1)}{2} \phi_{b}-\frac{(\sqrt{5}-1)}{2} \phi_{c}-\phi_{d}\right]\right| \vec{\mu} \\
& \times\left|\frac{1}{\sqrt{5-\sqrt{5}}}\left[\phi_{a}-\frac{(\sqrt{5}-1)}{2} \phi_{b}-\frac{(\sqrt{5}-1)}{2} \phi_{c}+\phi_{d}\right]\right\rangle,
\end{aligned}
$$

where

$$
\vec{\mu}=-e \vec{r}_{a}-e\left(\vec{r}_{b}+\vec{R}_{b a}\right)-e\left(\vec{r}_{c}+\vec{R}_{c a}\right)-e\left(\vec{r}_{d}+\vec{R}_{d a}\right)
$$

Noticing that $\left\langle\phi_{i}\left|\vec{\gamma}_{i}\right| \phi_{i}\right\rangle=0$ and ignoring two center integrals yields

$$
\begin{aligned}
\sqrt{2} \vec{\mu}_{23} & =\frac{-e \sqrt{2}}{5-\sqrt{5}}\left[-\frac{(\sqrt{5}-1)^{2}}{4} \vec{R}_{b a}+\frac{(\sqrt{5}-1)^{2}}{4} \vec{R}_{c a}-\vec{R}_{d a}\right] \\
& \approx \frac{-e \sqrt{2}}{5-\sqrt{5}}\left[-\frac{(\sqrt{5}-1)^{2}}{4} \vec{R}_{b a}+\frac{(\sqrt{5}-1)^{2}}{4} 2 \vec{R}_{b a}-3 \vec{R}_{b a}\right] \\
& =\frac{3+\sqrt{5}}{\sqrt{2}(5-\sqrt{5})}\left(e \vec{R}_{b a}\right)
\end{aligned}
$$

In similar fashion, we have

$$
\begin{aligned}
& \left\langle\Phi_{g}^{0}|\vec{\mu}| \Phi_{c_{i}}^{0}\right\rangle=\sqrt{2}\left\langle\chi_{+}|\vec{\mu}| \chi_{2}\right\rangle=\sqrt{2} \vec{\mu}_{b 2} \\
& =\sqrt{2}\left\langle\frac{1}{\sqrt{5-\sqrt{5}}}\left[\phi_{a}+\frac{(\sqrt{5}-1)}{2} \phi_{b}-\frac{(\sqrt{5}-1)}{2} \phi_{c}-\phi_{d}\right]\right| \vec{\mu} \\
& \times\left|\frac{1}{\sqrt{5+\sqrt{5}}}\left[\phi_{a}-\frac{(\sqrt{5}+1)}{2} \phi_{b}+\frac{(\sqrt{5}+1)}{2} \phi_{c}-\phi_{d}\right]\right\rangle, \\
& \left.=\frac{-e}{\sqrt{20}}\left[\frac{(5-1)}{4} \vec{R}_{b a}-\frac{(5-1)}{4} \vec{R}_{c a}-\vec{R}_{d a}\right)\right] \\
& =\frac{-e \sqrt{2}}{\sqrt{20}}\left[-\frac{(5-1)}{4} \vec{R}_{b a}-\frac{(5-1)}{4} \vec{R}_{c a}+\vec{R}_{d a}\right] \approx 0
\end{aligned}
$$

and

$$
\begin{aligned}
& \left\langle\Phi_{e_{1}}^{0}|\vec{\mu}| \Phi_{e_{2}}^{0}\right\rangle=\left\langle\chi_{3}|\vec{\mu}| \chi_{4}\right\rangle=\vec{\mu}_{34} \\
& =\left\langle\frac{1}{\sqrt{5-\sqrt{5}}}\left[\phi_{a}-\frac{(\sqrt{5}-1)}{2} \phi_{b}-\frac{(\sqrt{5}-1)}{2} \phi_{c}+\phi_{d}\right]\right| \vec{\mu} \\
& \quad \times\left|\frac{1}{\sqrt{5+\sqrt{5}}}\left[\phi_{a}-\frac{(\sqrt{5}+1)}{2} \phi_{b}+\frac{(\sqrt{5}+1)}{2} \phi_{c}-\phi_{d}\right]\right\rangle \\
& \left.=\frac{-e}{\sqrt{20}}\left[\frac{(5-1)}{4} \vec{R}_{b a}-\frac{(5-1)}{4} \vec{R}_{c a}-\vec{R}_{d a}\right)\right] \approx \frac{2}{\sqrt{5}}\left(e \vec{R}_{b a}\right)
\end{aligned}
$$

Appendix C. Calculation of $\left\langle\chi_{3}|V| \chi_{2}\right\rangle, \sqrt{2}\left\langle\chi_{4}|V| \chi_{2}\right\rangle$ and $\left\langle\chi_{3}|V| \chi_{4}\right\rangle$

We shall treat a non-polar molecule dissolved in polar molecules. In this case, we have

$$
\begin{aligned}
& \left\langle\Phi_{e_{1}}^{0}|V| \Phi_{g}^{0}\right\rangle=\sqrt{2}\left\langle\chi_{3}|V| \chi_{2}\right\rangle \\
& =\sum_{0}\left[\frac{\left(\vec{m}_{\alpha} \cdot \sqrt{2} \vec{\mu}_{32}\right)}{R_{0}^{3}}-\frac{3\left(\vec{m}_{\alpha} \cdot \vec{R}_{o}\right)\left(\vec{R}_{0} \cdot \sqrt{2} \vec{\mu}_{32}\right)}{R_{\alpha}^{5}}\right] \\
& =\frac{3+\sqrt{5}}{\sqrt{2}(5-\sqrt{5})} \sum_{0}\left[\frac{\vec{m}_{0} \cdot\left(e \vec{R}_{b a}\right)}{R_{\alpha}^{3}}-\frac{3\left(\vec{m}_{o} \cdot \vec{R}_{0}\right)\left\{\vec{R}_{\alpha} \cdot\left(e \vec{R}_{b a}\right)\right\}}{R_{\alpha}^{5}}\right] \\
& \approx \frac{3+\sqrt{5}}{\sqrt{2}(5-\sqrt{5})} \sum_{0} V_{\dot{m}_{o}}\left(R_{\alpha}\right)
\end{aligned}
$$




$$
\begin{aligned}
& \left\langle\Phi_{e_{2}}^{0}|V| \Phi_{g}^{0}\right\rangle=\sqrt{2}\left\langle\chi_{4}|V| \chi_{2}\right\rangle \\
& =\sum_{0}\left[\frac{\left(\vec{m}_{o} \cdot \sqrt{2} \vec{\mu}_{42}\right)}{R_{0}^{3}}-\frac{3\left(\vec{m}_{\alpha} \cdot \vec{R}_{\alpha}\right)\left(\vec{R}_{0} \cdot \sqrt{2} \vec{\mu}_{42}\right)}{R_{\alpha}^{5}}\right] \approx 0 \\
& \left\langle\Phi_{e_{1}}^{0}|V| \Phi_{e_{1}}^{0}\right\rangle=\left\langle\chi_{3}|V| \chi_{4}\right\rangle \\
& =\sum_{0}\left[\frac{\left(\vec{m}_{0} \cdot \vec{\mu}_{34}\right)}{R_{0}^{3}}-\frac{3\left(\vec{m}_{\alpha} \cdot \vec{R}_{\alpha}\right)\left(\vec{R}_{\alpha} \cdot \sqrt{2} \vec{\mu}_{34}\right)}{R_{\alpha}^{5}}\right] \\
& \approx \frac{2}{\sqrt{5}} \sum_{\alpha} V_{\vec{m}_{c}}\left(R_{\alpha}\right)
\end{aligned}
$$

where

$$
V_{\vec{m}_{\mathrm{o}}}\left(R_{\alpha}\right)=\left[\frac{\vec{m}_{0} \cdot\left(e \vec{R}_{b a}\right)}{R_{0}^{3}}-\frac{3\left(\vec{m}_{\alpha} \cdot \vec{R}_{\alpha}\right)\left\{\vec{R}_{0} \cdot\left(e \vec{R}_{b a}\right)\right\}}{R_{\alpha}^{5}}\right](\mathrm{C}-4)
$$

For a non-polar molecule dissolved in a non-polar crystal, we have

$$
\begin{aligned}
\left\langle\Phi_{e_{1}}^{0}|V| \Phi_{g}^{0}\right\rangle & =\sqrt{2}\left\langle\chi_{3}|V| \chi_{2}\right\rangle \\
& \approx-\sum_{D} \frac{2 \alpha_{D}\left(\vec{R}_{D A} \cdot \sqrt{2} \vec{\mu}_{32}\right)}{R_{D A}^{6}} \\
& =-\frac{3+\sqrt{5}}{\sqrt{2}(5-\sqrt{5})} \sum_{D} \frac{2 \alpha_{D}\left\{\vec{R}_{D A} \cdot\left(e \vec{R}_{b a}\right)\right\}}{R_{D A}^{6}} \\
& =-\frac{3+\sqrt{5}}{\sqrt{2}(5-\sqrt{5})} \sum_{D} V_{\alpha_{A}}\left(\vec{R}_{D_{A}}\right) \\
\left\langle\Phi_{e_{2}}^{0}|V| \Phi_{g}^{0}\right\rangle & =\sqrt{2}\left\langle\chi_{4}|V| \chi_{2}\right\rangle \approx 0 \\
\left\langle\Phi_{e_{1}}^{0}|V| \Phi_{e_{2}}^{0}\right\rangle & =\left\langle\chi_{3}|V| \chi_{4}\right\rangle \approx-\frac{2}{\sqrt{5}} \sum_{\alpha} V_{\mathrm{o}_{D}}\left(\vec{R}_{D A}\right)(\mathrm{C}-7)
\end{aligned}
$$

where

$$
V_{\alpha_{D}}\left(\vec{R}_{D A}\right)=\frac{2 \alpha_{D}\left\{\vec{R}_{D A} \cdot\left(e \vec{R}_{b a}\right)\right\}}{R_{D A}^{6}}
$$

\section{REFERENCES}

1. Moerner, W. E.; Kador, L. Phys. Rev. Lett. 1989, 62, 2535.

2. Jung, J. A.; Schlittler, R. R.; Ginzawski, J. K.; Tang, H.; Joachim, C. Science 1996, 271, 281.

3. Betzig, E.; Chichester, R. J. Science 1993, 262, 142.

4. Eiger, M.; Rigler, R. Proc. Natl. Acad. Sci. 1994, 91, 5740.

5. Orrit, M.; Bernhard, J. Phys. Rev. Lett. 1990, 65, 2716.

6. Wild, U. P.; Güttler, F.; Pirotta, M.; Renn, A. Chem. Phys. Lett. 1992, 193, 451.

7. Basche, T.; Moerner, W. E.; Orrit, M.; Wild, U. P. eds., "Single Molecule Optical Detection, Imaging and Spectroscopy" (VCH, Weinheim, 1997).

8. Moerner, W. E. Acct. Chem. Res. 1996, 29, 563.

9. Wild, W. P.; Güttler, F.; Pirotta, M.; Renn, A. J. Lumin. 1994, 1003, 60-61.

10. Orrit, M.; Bernard, J.; Zumbusch, A.; Dersnor, R. Chem. Phys. Lett. 1992, 196, 595.

11. Pirotta, M. PhD. Thesis, ETH Z ich, Diss. 1998, No. 11887.

12. Bach, H. PhD. Thesis, ETH Z ich, Diss. 1998, No. 12934.

13. Brunel, C.; Tamarat, P.; Louinis, B.; Woehl, J. C.; Orrit, M. J. Phys. Chem. 1999, A103, 2429.

14. Gaussian 98 (Revision A.7), Frisch, M. J.; Trucks, G. W.; Schlegel, H. B.; Scuseria, G. E.; Robb, M. A.; Cheeseman, J. R.; Zakrzewski, V. G.; Montgomery, Jr., J. A.; Stratmann, R. E.; Burant, J. C.; Dapprich, S.; Millam, J. M.; Daniels, A. D.; Kudin, K. N.; Strain, M. C.; Farkas, O.; Tomasi, J.; Barone, V.; Cossi, M.; Cammi, R.; Mennucci, B.; Pomelli, C.; Adamo, C.; Clifford, S.; Ochterski, J.; Petersson, G. A.; Ayala, P. Y.; Cui, Q.; Morokuma, K.; Malick, D. K.; Rabuck, A. D.; Raghavachari, K.; Foresman, J. B.; Cioslowski, J.; Ortiz, J. V.; Baboul, A. G.; Stefanov, B. B.; Liu, G.; Liashenko, A.; Piskorz, P.; Komaromi, I.; Gomperts, R.; Martin, R. L.; Fox, D. J.; Keith, T.; Al-Laham, M. A.; Peng, C. Y.; Nanayakkara, A.; Gonzalez, C.; Challacombe, M.; Gill, P. M. W.; Johnson, B. G.; Chen, W.; Wong, M. W.; Andres, J. L.; Head-Gordon, M.; Replogle E. S. and Pople, J. A. Gaussian, Inc., Pittsburgh PA, 1998. 